\title{
Flexible Allocation of Capacity in Multi-Cell CDMA Networks
}

Robert Akl, Manju Hegde,

Mort Naraghi-Pour*, Paul Min

Washington University, St. Louis, MO

*Louisiana State University, Baton Rouge, LA 


\section{Outline}

- Capacity and Probability of Outage

- Calculation of inter-cell interference

- Capacity region

- Power compensation factor

- Effects of different cell sizes

- Optimization of capacity

- Flexible allocation of capacity

- Results 
Nine Cell Network

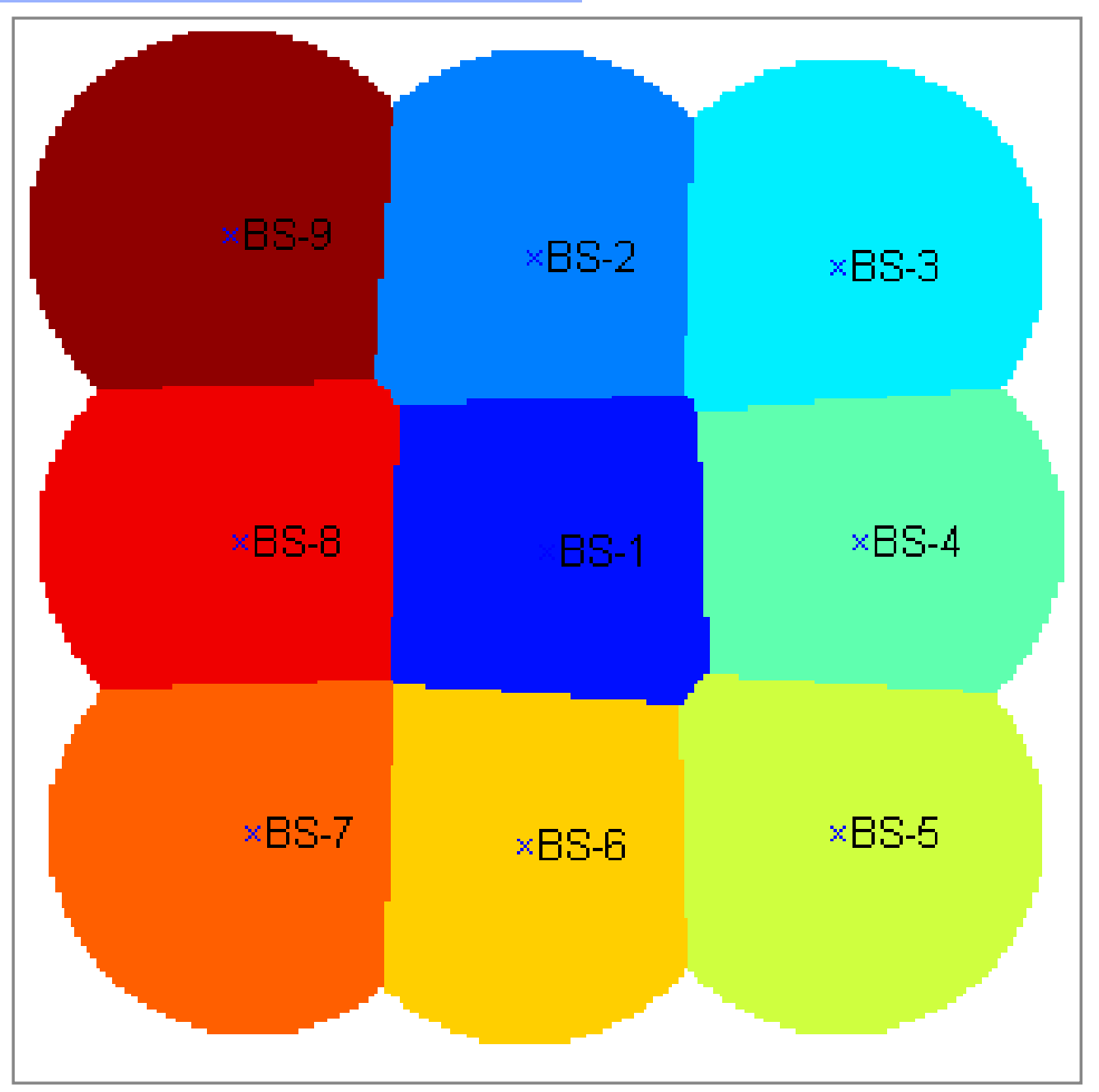




\section{Probability of Outage For Each Cell}

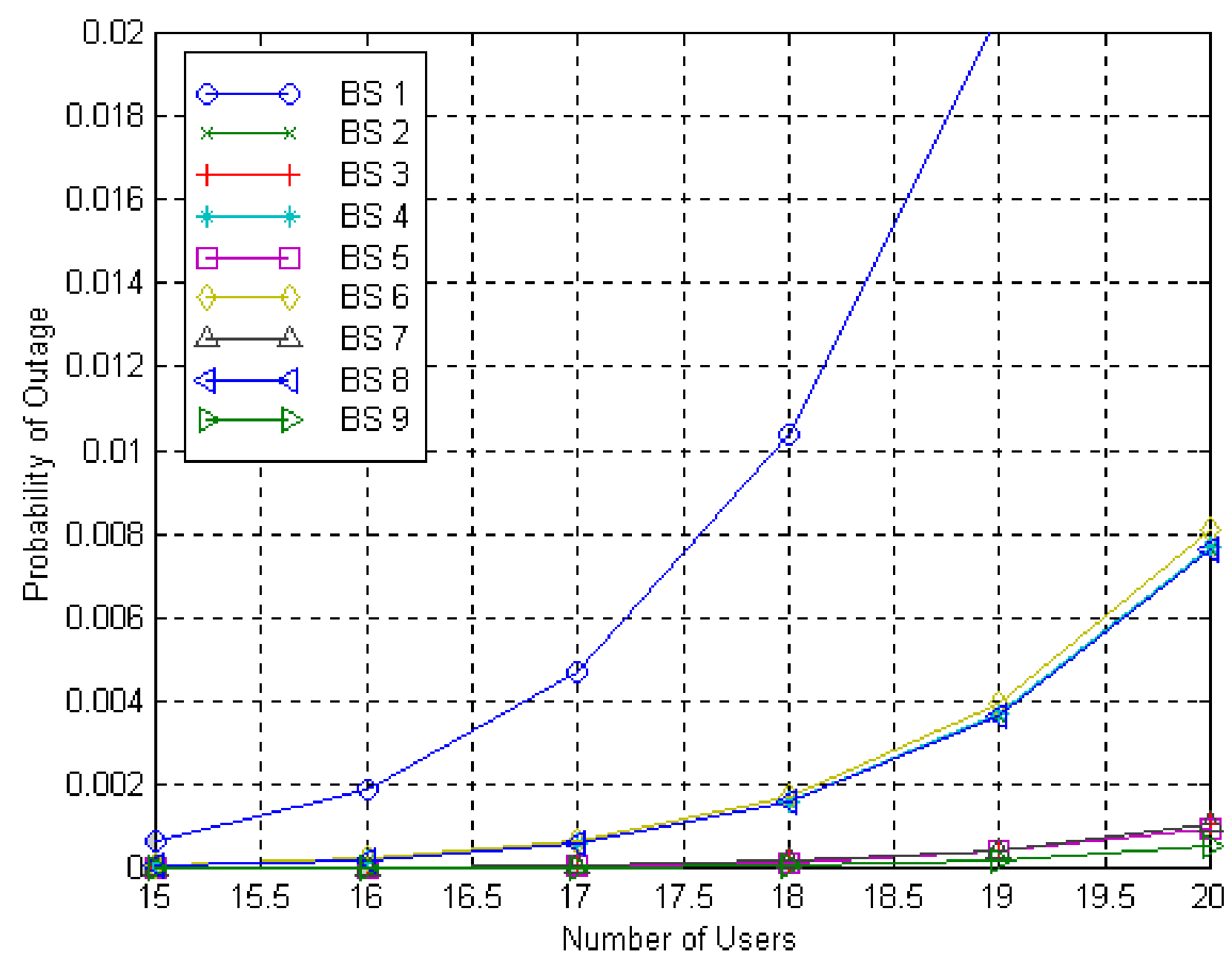




\section{Probability of Outage for Single Cell}

$P_{\mathrm{out}}^{i}=\operatorname{Pr}\left(\sum_{l=2}^{n_{i}} v_{l}>\frac{(W / R)(1-\eta)^{\Delta}}{E_{b} / I_{o}}=K_{o}^{\prime}\right)$

$\frac{I_{o}}{N_{o}}<\frac{1}{\eta}$ where $\eta<1$,

$\operatorname{Pr}\left(v_{l}=1\right)=\alpha$, the voice activity factor,

$n_{i}$ is the number of users in cell $i$. 


\section{Probability of Outage for Single Cell}

$$
\begin{aligned}
& P_{\text {out }}^{i}=\operatorname{Pr}\left(\sum_{l=2}^{n_{i}} v_{l}>K_{o}^{\prime}\right)<\operatorname{Pr}\left(\sum_{l=1}^{n_{i}} v_{l}>K_{o}^{\prime}\right) \\
& \text { Let } Z_{i} \stackrel{\Delta}{=} \sum_{l=1}^{n_{i}} v_{l} \\
& \text { where } \operatorname{Pr}\left(n_{i}=k\right)=\frac{\left(\lambda_{i} / \mu_{i}\right)^{k}}{k !} e^{-\lambda_{i} / \mu_{i}}, k=0,1,2, \ldots \\
& P_{\text {out }}^{i}<\operatorname{Pr}\left(Z_{i}>K_{o}^{\prime}\right)=e^{-\alpha \lambda_{i} / \mu_{i}} \sum_{k=\left[K_{o}^{\prime}\right.}^{\infty}\left(\alpha \lambda_{i} \mu_{i}\right)^{k} / k !
\end{aligned}
$$




\section{Gaussian Approximation}

- We approximate the Poisson by a Gaussian variable with the same mean and variance:

$$
\begin{aligned}
& P_{\text {out }}^{i} \approx Q\left(\frac{K_{o}^{\prime}-\mathrm{E}\left(Z_{i}\right)}{\sqrt{\operatorname{var}\left(Z_{i}\right)}}\right)=Q\left(\frac{K_{o}^{\prime}-\alpha \lambda_{i} / \mu_{i}}{\sqrt{\alpha \lambda_{i} / \mu_{i}}}\right) \\
& Q(y)=\int_{y}^{\infty} \frac{1}{\sqrt{2 \pi}} e^{-x^{2} / 2} d x
\end{aligned}
$$




\section{Probability of Outage for Multiple Cells}

$\boldsymbol{l}_{j i}$ : Inter-cell interference from cell $j$ to cell
$i$.

$P_{\text {out }}^{i}=\operatorname{Pr}\left(\sum_{l=1}^{n_{i}+\sum_{j=1}^{M} I_{j i}} v_{l}>\frac{(W / R)(1-\eta)}{E_{b} / I_{o}}\right)$

$M$ is the total number of cells. 


\section{Shadow and Rayleigh Fading}

- Assume power control overcomes both large scale path loss and shadow fading, but not Rayleigh fading.

- The average of the Rayleigh fading is the shadow fading on that path. 


\section{Inter-Cell Interference}

$$
I_{j i}=\mathrm{E} \iint_{\text {cell } j} \frac{r_{j}^{m}(x, y) 10^{\varsigma_{j} / 10}}{r_{i}^{m}(x, y) / X_{i}^{2}} \rho_{j} d A(x, y)
$$

$m$ is the path loss exponent.

$\varsigma_{i}$ is the decibel attenuation due to shadowing, and has zero mean and standard deviation $\sigma_{s}$. $\mathrm{E}\left[X_{i}^{2} \mid \varsigma_{i}\right]=10^{-\varsigma_{i} / 10}$

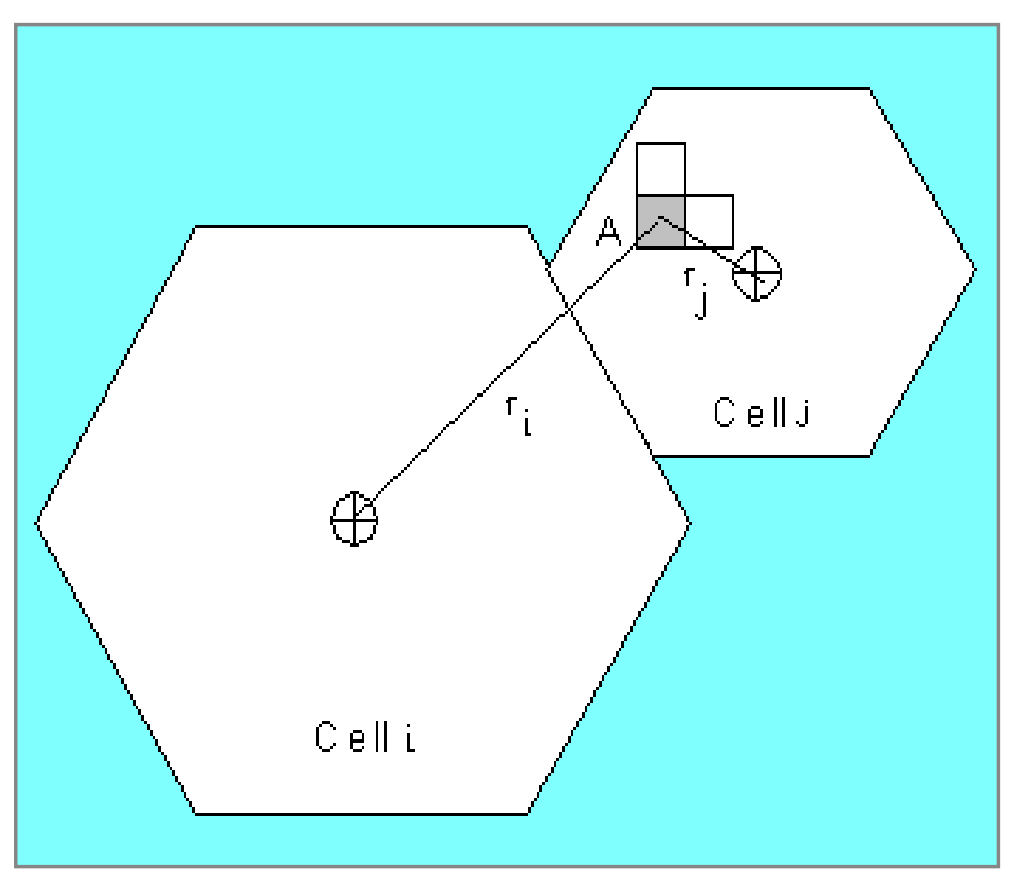

$\rho_{j}=\frac{n_{j}}{\text { Area of cell } j}$ 


\section{Soft Handoff}

- User is permitted to be in soft handoff to its two nearest cells.

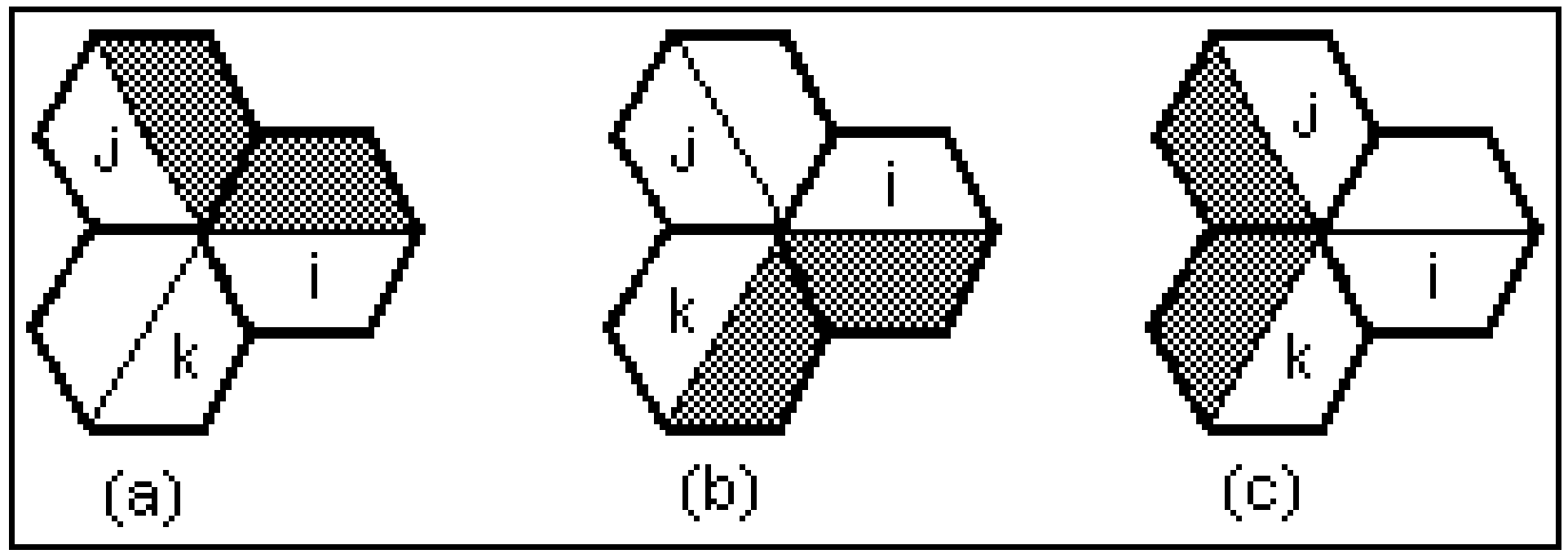




\section{Soft Handoff}

$$
\begin{aligned}
& I_{j i}=\iint_{\text {region (a) }} \frac{r_{j}^{m}}{r_{i}^{m}} \mathrm{E}\left[10^{\varsigma_{j} / 10} X_{i}^{2} \mid r_{j}^{m} 10^{\varsigma_{j} / 10}<r_{i}^{m} 10^{\varsigma_{i} / 10}\right] \rho d A(x, y) \\
& I_{k i}=\iint_{\text {region(b) }} \frac{r_{k}^{m}}{r_{i}^{m}} \mathrm{E}\left[10^{\varsigma_{k} / 10} X_{i}^{2} \mid r_{k}^{m} 10^{\varsigma_{k} / 10}<r_{i}^{m} 10^{\varsigma_{i} / 10}\right] \rho d A(x, y) \\
& I_{j i}=\iint_{\text {region(c) }} \frac{r_{j}^{m}}{r_{i}^{m}} \mathrm{E}\left[10^{\varsigma_{j} / 10} X_{i}^{2} \mid r_{j}^{m} 10^{\varsigma_{j} / 10}<r_{k}^{m} 10^{\varsigma_{k} / 10}\right] \rho d A(x, y) \\
& I_{k i}=\iint_{\text {region(c) }} \frac{r_{k}^{m}}{r_{i}^{m}} \mathrm{E}\left[10^{\varsigma_{k} / 10} X_{i}^{2} \mid r_{k}^{m} 10^{\varsigma_{k} / 10}<r_{j}^{m} 10^{\varsigma_{j} / 10}\right] \rho d A(x, y)
\end{aligned}
$$




\section{Inter-Cell Interference Factor}

- $K_{j i}$ : per user inter-cell interference factor from cell $j$ to cell $i$

- $n_{j}$ users in cell $j$ produce an amount of interference in cell $i$ equal to $n_{j} K_{j i}$

- $K_{i i}$ are not necessarily zero because of soft handoff, users in cell $i$ can cause inter-cell interference to cell $i$. 


\section{Capacity Region}

$$
\frac{E_{b} / N_{o}}{\alpha\left(E_{b} / N_{o}\right)\left(n_{i}-1+\sum_{j=1}^{M} n_{j} \kappa_{j i}\right) /(W / R)+1} \geq\left(\frac{E_{b}}{I_{o}}\right)_{\text {req }}
$$

for $i=1, \ldots, M$.

$$
\begin{gathered}
n_{i}+\sum_{j=1}^{M} n_{j} \kappa_{j i} \leq \frac{W / R}{\alpha}\left(\frac{1}{E_{b} / I_{o}}-\frac{1}{E_{b} / N_{o}}\right)+1 \stackrel{\Delta}{=} c_{e f f} \\
\text { for } i=1, \ldots, M .
\end{gathered}
$$




\section{Capacity Region}

- Capacity region: set of all feasible user configurations

- Uniform capacity: $n_{i}=c_{1}$ for all $i$

- Two level capacity: $m_{1}$ cells have capacity $c_{1}$ and $m_{2}$ cells have capacity $c_{2}$ where $m_{1}$ $+m_{2}=M$ 


\section{Example: 2 Cell Network}

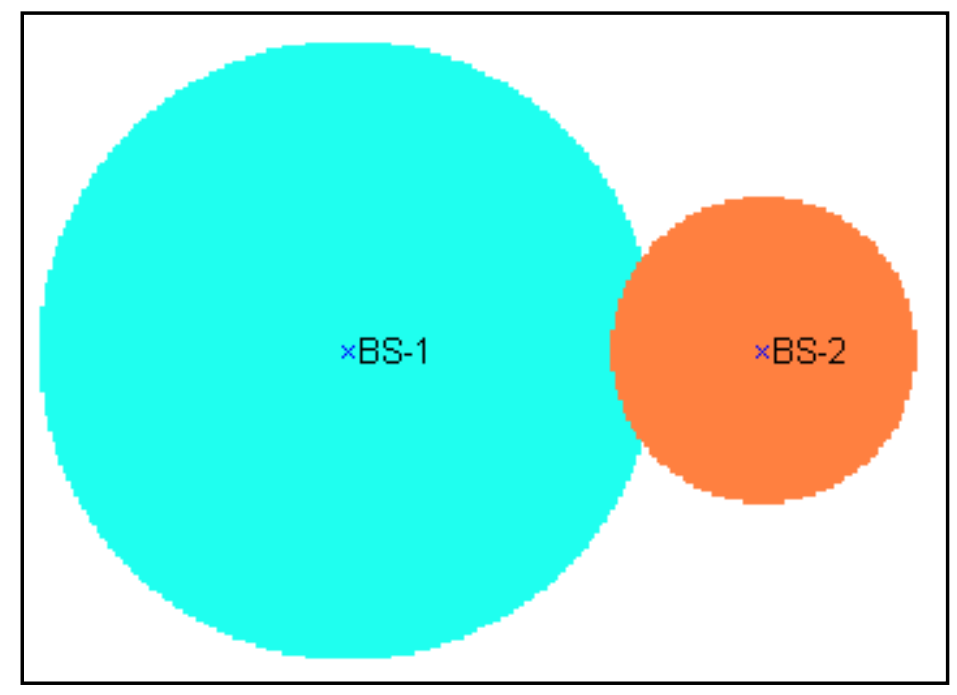

\begin{tabular}{|c|c|c|}
\hline$K_{i j}$ & BS-1 & BS-2 \\
\hline BS-1 & 0 & 4.0130 \\
\hline BS-2 & 0.0617 & 0 \\
\hline
\end{tabular}




\section{Capacity Region}

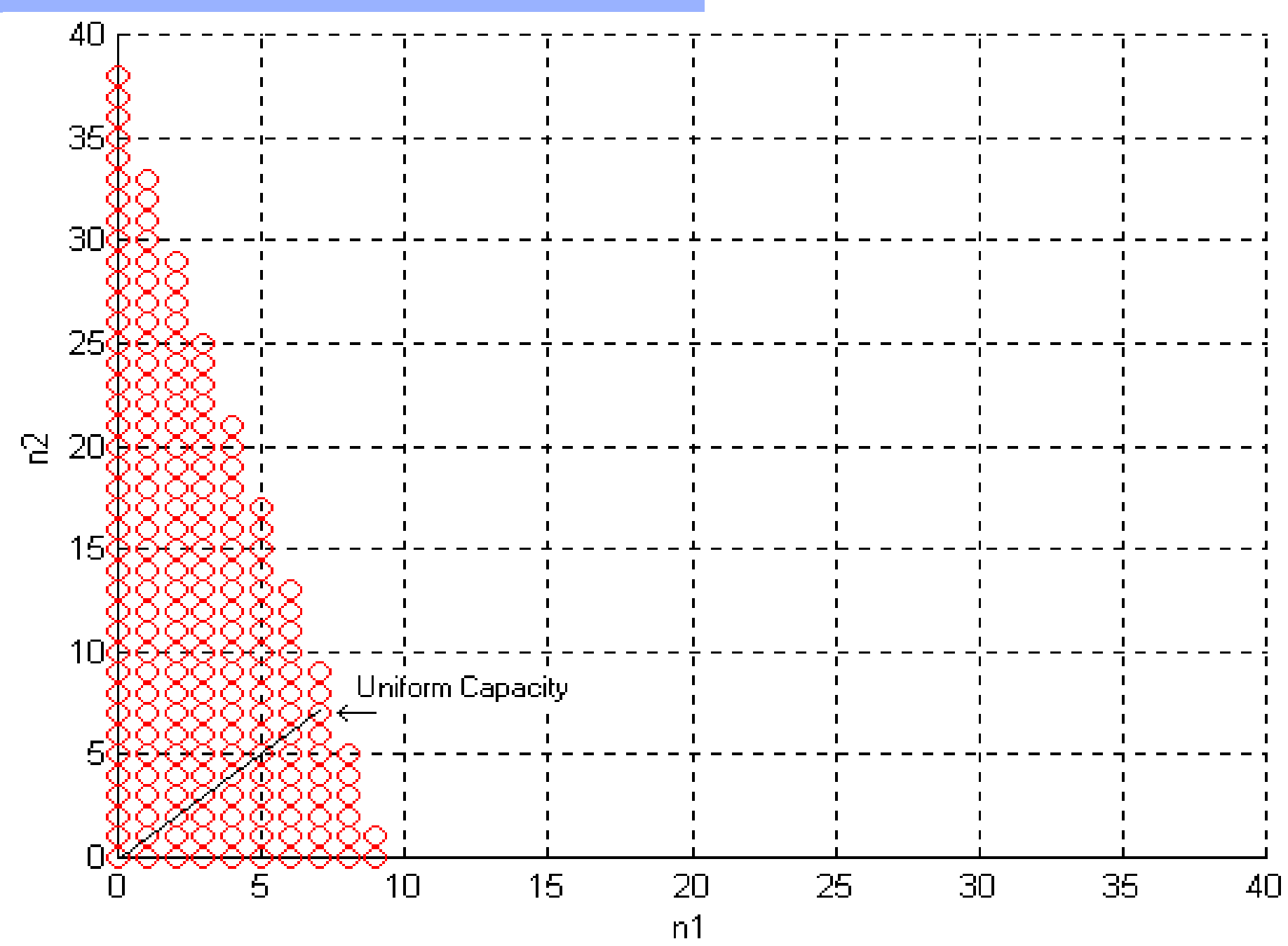




\section{Power Compensation Factor}

- Fine tune the nominal power of the users

- PCF defined for each cell

- PCF is a design tool to maximize the capacity of the entire network 


\section{Power Compensation Factor}

- Interference is linear in PCF

$$
\begin{aligned}
& I_{j i}=\iint_{\text {cell } j} \frac{\beta_{j} r_{j}^{m} 10^{\varsigma_{j} / 10}}{r_{i}^{m} / X_{i}^{2}} \rho_{j} d A \\
& n_{i}+\sum_{j=1}^{M} n_{j} \frac{\beta_{j} \kappa_{j i}}{\beta_{i}} \leq c_{e f f} \text { for } i=1, \ldots, M .
\end{aligned}
$$




\section{Sensitivity Analysis}

- Derivative of capacity function with respect to the PCF

- Capture effect of increases in PCF in one cell on the capacity of whole network

- Tool to flexibly distribute capacity between cells 


\section{Sensitivity Analysis}

- For uniform capacity case:

$c_{1}=\min _{i}\left[\frac{c_{e f f}}{1+\frac{1}{\beta_{i}} \sum_{j=1}^{M} \beta_{j} \kappa_{j i}}\right]$ for $i=1, \ldots, M$.

Let $i^{*}$ be the index that minimizes the above equation.

The derivative $\frac{d c_{1}}{d \beta_{k}}$ can be calculated . 


\section{Sensitivity Analysis}

$\frac{d c_{1}}{d \beta_{k}}=\frac{-c_{e f f} \beta_{i^{*}} \kappa_{k i^{*}}}{\left(\beta_{i^{*}}+\sum_{j=1}^{M} \beta_{j} \kappa_{j i^{*}}\right)^{2}}$ if $k \neq i^{*}$

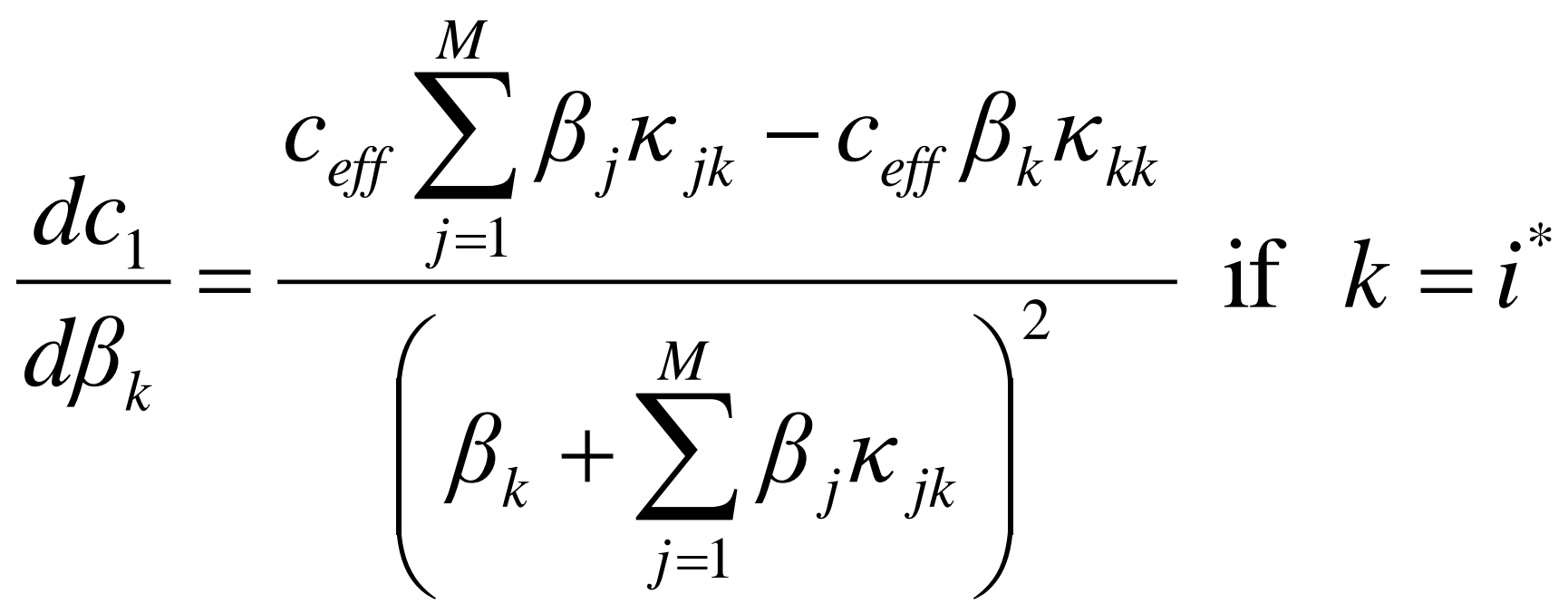




\section{Optimization}

- Optimize the sum capacity: the sum of the capacities of the cells

- Constraint: PCF between a min and a max

- Use the derivatives in steepest descent algorithm

- New PCF is the factor that the nominal power needs to be increased by for every cell

- Each PCF is used by its Base Station in the Closed Loop Power Control 


\section{Optimization of Uniform Capacity}

$\max _{\bar{\beta}, c_{1}} \quad M c_{1}, \quad$ (sum capacity)

subject to

$$
\begin{aligned}
& 1 \leq \bar{\beta} \leq \bar{\beta}^{\max } \\
& c_{1}+\sum_{j=1}^{M} c_{1} \frac{\beta_{j} \kappa_{j i}}{\beta_{i}} \leq c_{e f f}, \\
& \text { for } i=1, \ldots, M
\end{aligned}
$$




\section{Optimization of Two-Level Capacity}

$\max _{\bar{\beta}, c_{1}, c_{2}} \quad m_{1} c_{1}+m_{2} c_{2}, \quad$ (sum capacity)

subject to

$$
\begin{aligned}
& 1 \leq \bar{\beta} \leq \bar{\beta}^{\max }, \\
& n_{i}+\sum_{j=1}^{M} n_{j} \frac{\beta_{j} \kappa_{j i}}{\beta_{i}} \leq c_{e f f}, \text { for } i=1, \ldots, M, \\
& n_{i}=c_{1} \text { for } i=1, \ldots, m_{1}, \\
& n_{i}=c_{2} \text { for } i=m_{1}+1, \ldots, m_{1}+m_{2}, \\
& m_{1}+m_{2}=M .
\end{aligned}
$$




\section{Capacity Region}

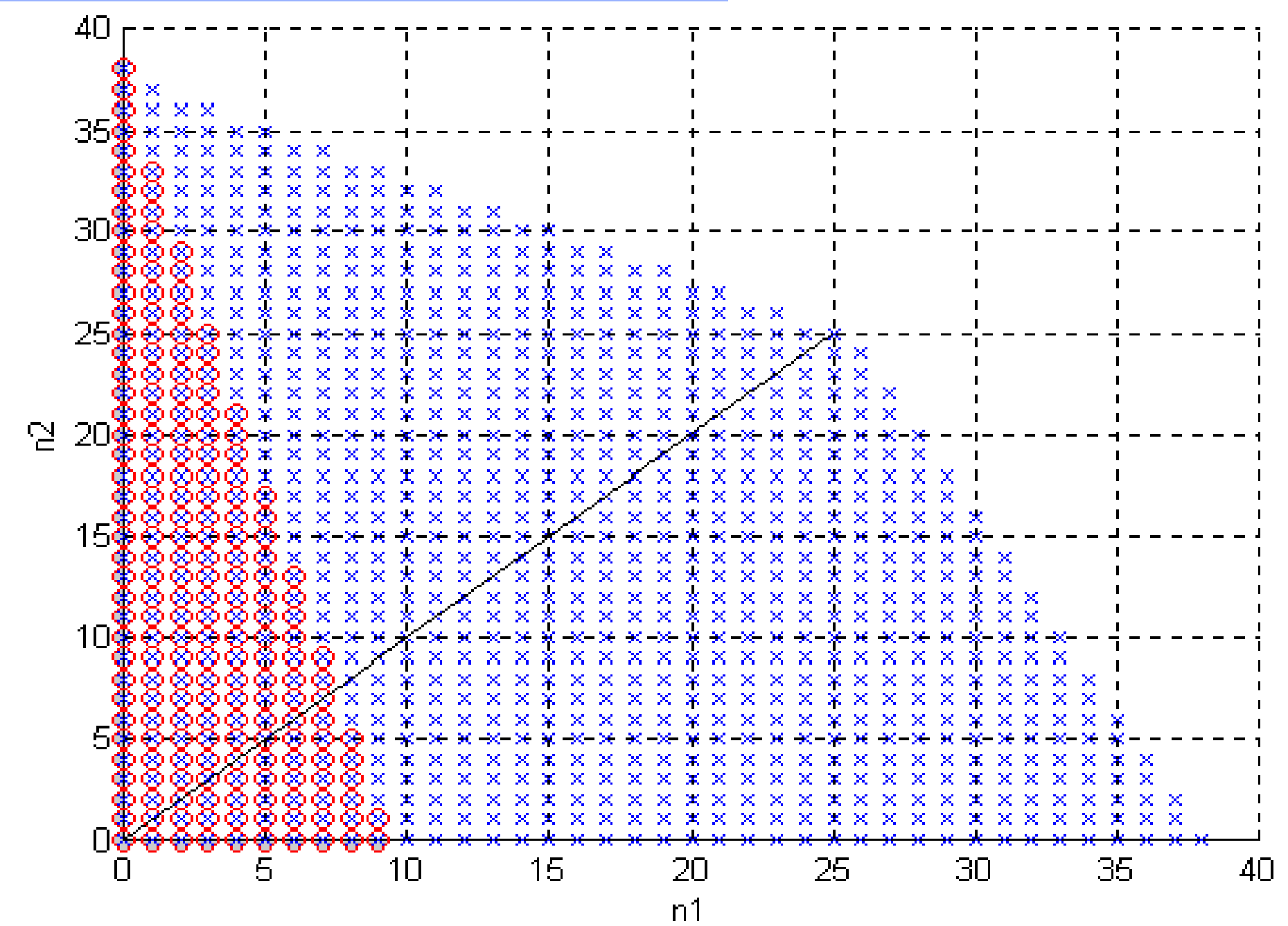




\section{Hard Handoff vs. Soft Handoff}

\begin{tabular}{|c|c|c|c|c|c|c|}
\hline HH & BS-1 & BS-2 & BS-3 & Cap & Opt & PCF \\
\hline BS-1 & 0 & 0.330 & 3.573 & 4 & 17 & 1.00 \\
\hline BS-2 & 0.331 & 0 & 3.420 & 4 & 17 & 1.00 \\
\hline BS-3 & 0.076 & 0.072 & 0 & 4 & 17 & 4.53 \\
\hline
\end{tabular}

\begin{tabular}{|c|c|c|c|c|c|c|}
\hline SH & BS-1 & BS-2 & BS-3 & Cap & Opt & PCF \\
\hline BS-1 & 0.106 & 0.116 & 0.603 & 17 & 26 & 1.00 \\
\hline BS-2 & 0.117 & 0.099 & 0.591 & 17 & 26 & 1.00 \\
\hline BS-3 & 0.066 & 0.063 & 0.006 & 17 & 26 & 2.56 \\
\hline
\end{tabular}

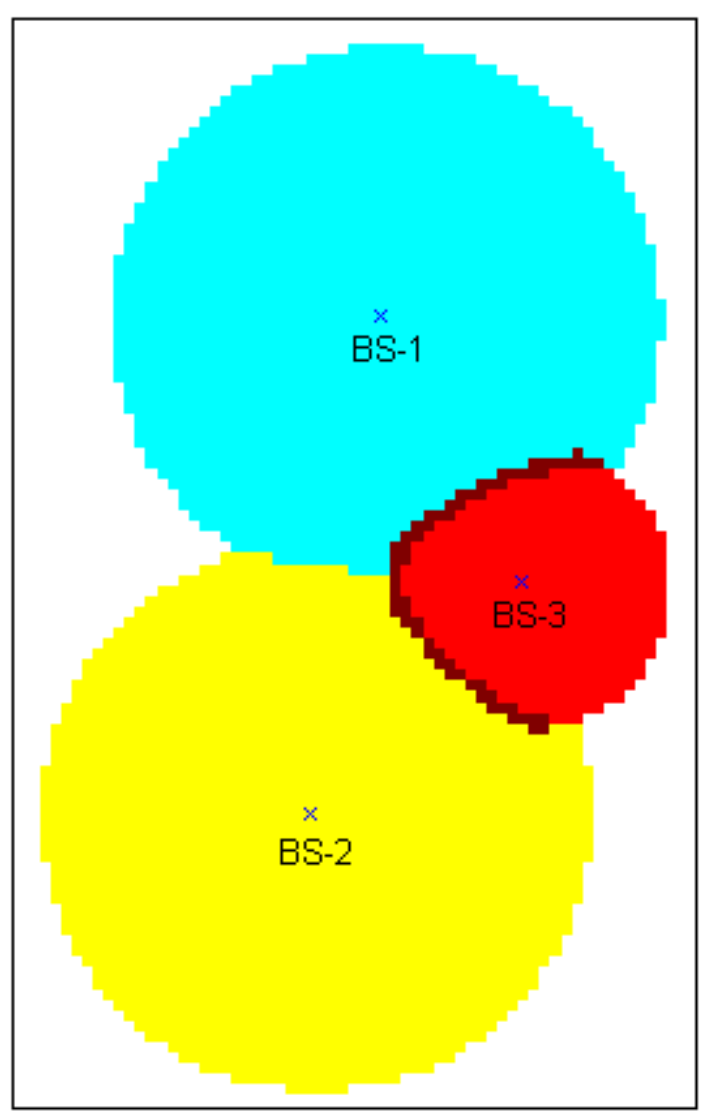




\section{Seven Cell Network}

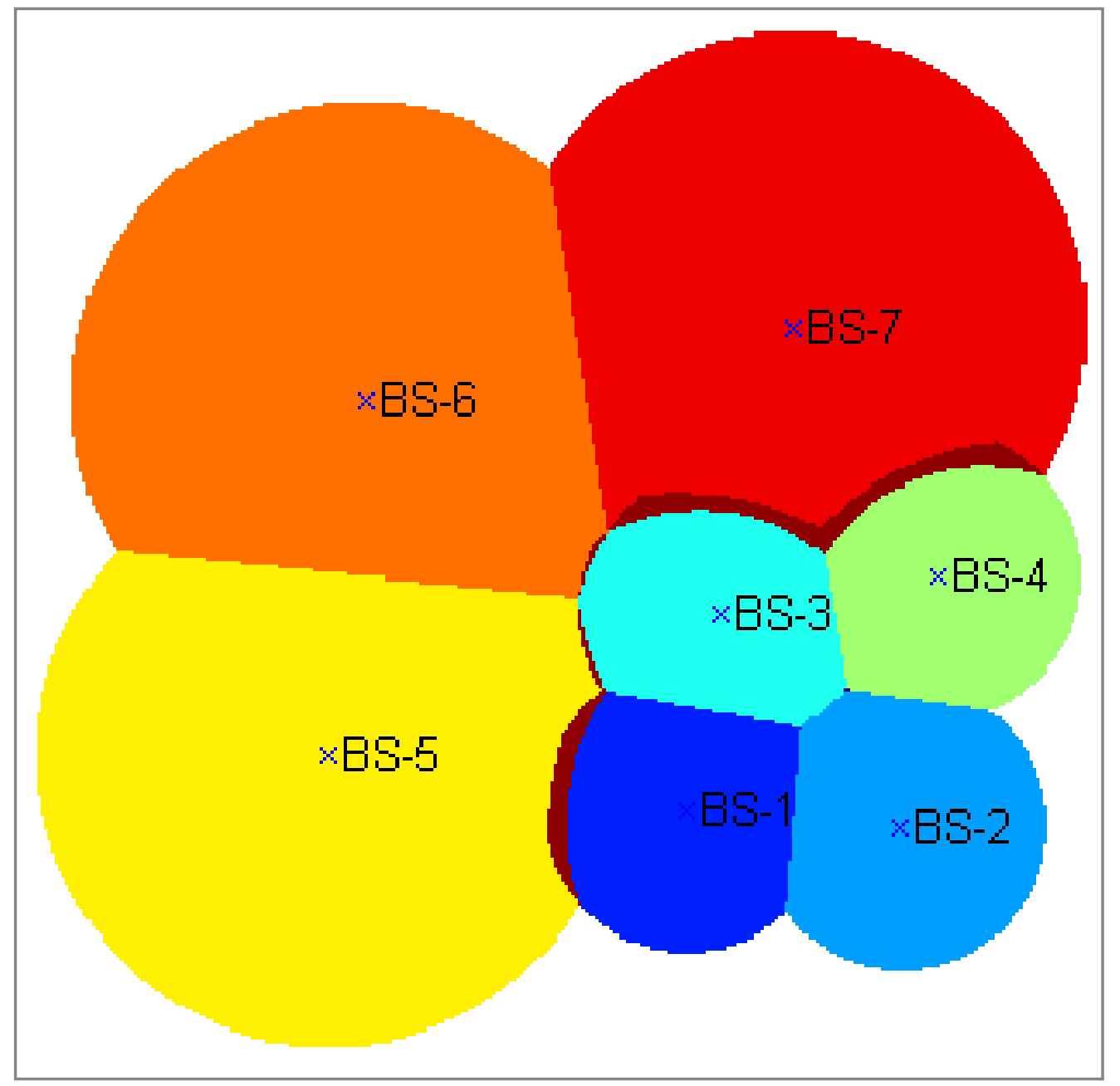




\section{Probability of Outage For Each Cell}

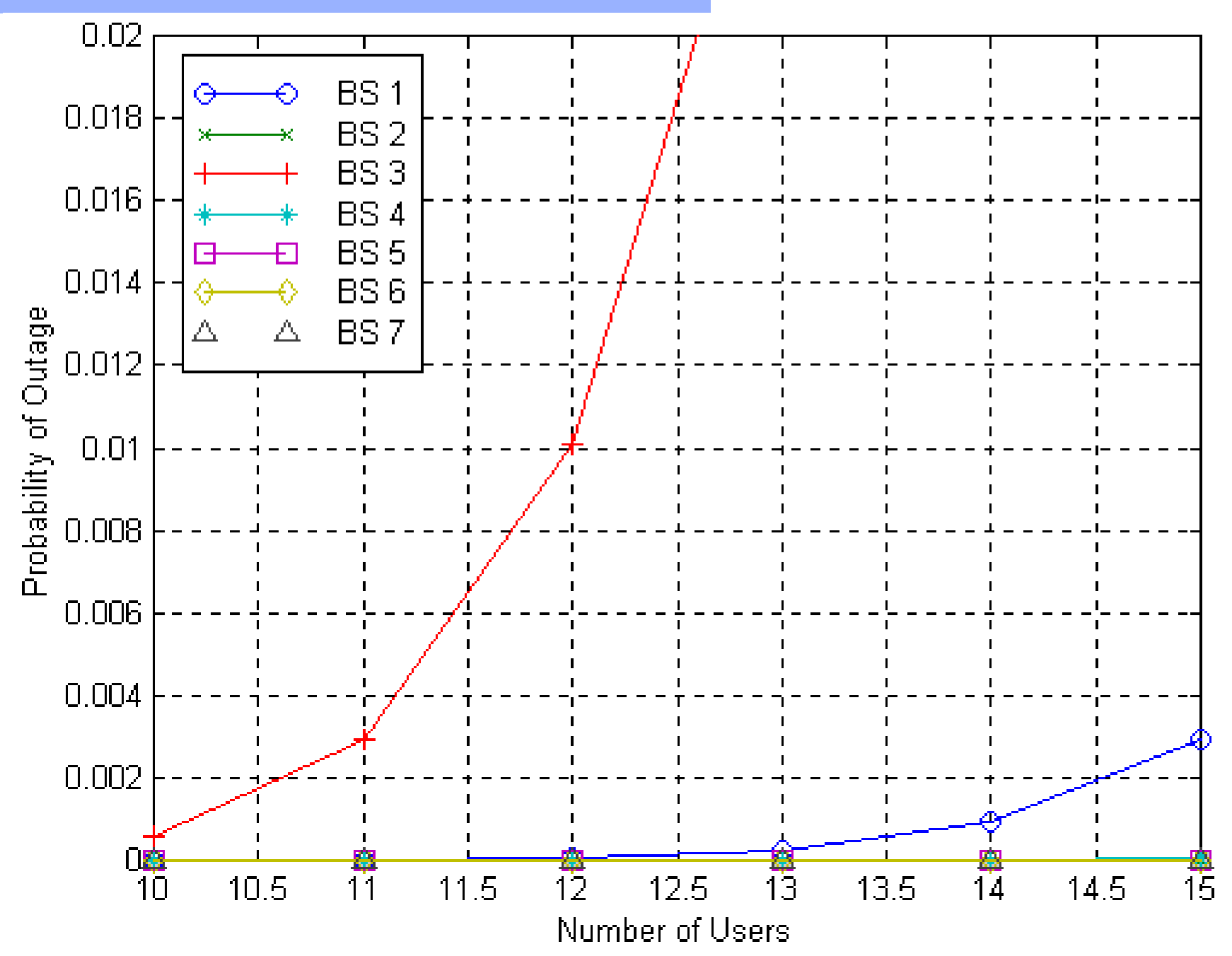




\section{Optimization}

- Uniform capacity

- C1: 11 (PCF=1) to 20 (PCF Optimized)

- Sum capacity: 77 to 140

- Two-level capacity

- Small cells: 14 (PCF=1) to 22 (PCF Optimized)

- Large cells: 7 (PCF=1) to 11 (PCF Optimized)

- Sum capacity: 77 to 121 


\section{Flexibility in Capacity Allocation}

- Case 1: $\max _{\bar{\beta}, n_{i}} \quad \sum_{i=1}^{M} n_{i}, \quad$ (sum capacity)

$$
\begin{array}{ll}
\text { subject to } & 1 \leq \bar{\beta} \leq \bar{\beta}^{\max }, \\
& n_{i}+\sum_{j=1}^{M} n_{j} \frac{\beta_{j} \kappa_{j i}}{\beta_{i}} \leq c_{e f f}, \\
& n_{i} \geq 20, \\
& \text { for } i=1, \ldots, M .
\end{array}
$$

- Result: sum capacity of 150. 


\section{Flexibility in Capacity Allocation}

- Case 2:

$$
\begin{array}{cl}
\max _{\bar{\beta}, n_{i}} & \sum_{i=1}^{M} n_{i}, \quad \text { (sum capacity) } \\
\text { subject to } & 1 \leq \bar{\beta} \leq \bar{\beta}^{\max } \\
& n_{i}+\sum_{j=1}^{M} n_{j} \frac{\beta_{j} \kappa_{j i}}{\beta_{i}} \leq c_{e f f}, \\
& \text { for } i=1, \ldots, M
\end{array}
$$

- Result: sum capacity of 152 but capacity of cell three drops from 20 to 13. 


\section{Flexibility in Capacity Allocation}

- Case 3:

$$
\begin{aligned}
\max _{\bar{\beta}, n_{i}} & \sum_{i=1}^{M} n_{i}, \quad \text { (sum capacity) } \\
\text { subject to } & 1 \leq \bar{\beta} \leq \bar{\beta}^{\max } \\
& n_{i}+\sum_{j=1}^{M} n_{j} \frac{\beta_{j} \kappa_{j i}}{\beta_{i}} \leq c_{e f f} \\
& \text { for } i=1, \ldots, M \\
& n_{3} \geq 25
\end{aligned}
$$

- Result: cell three has a capacity of 25 but sum capacity drops to 143 . 


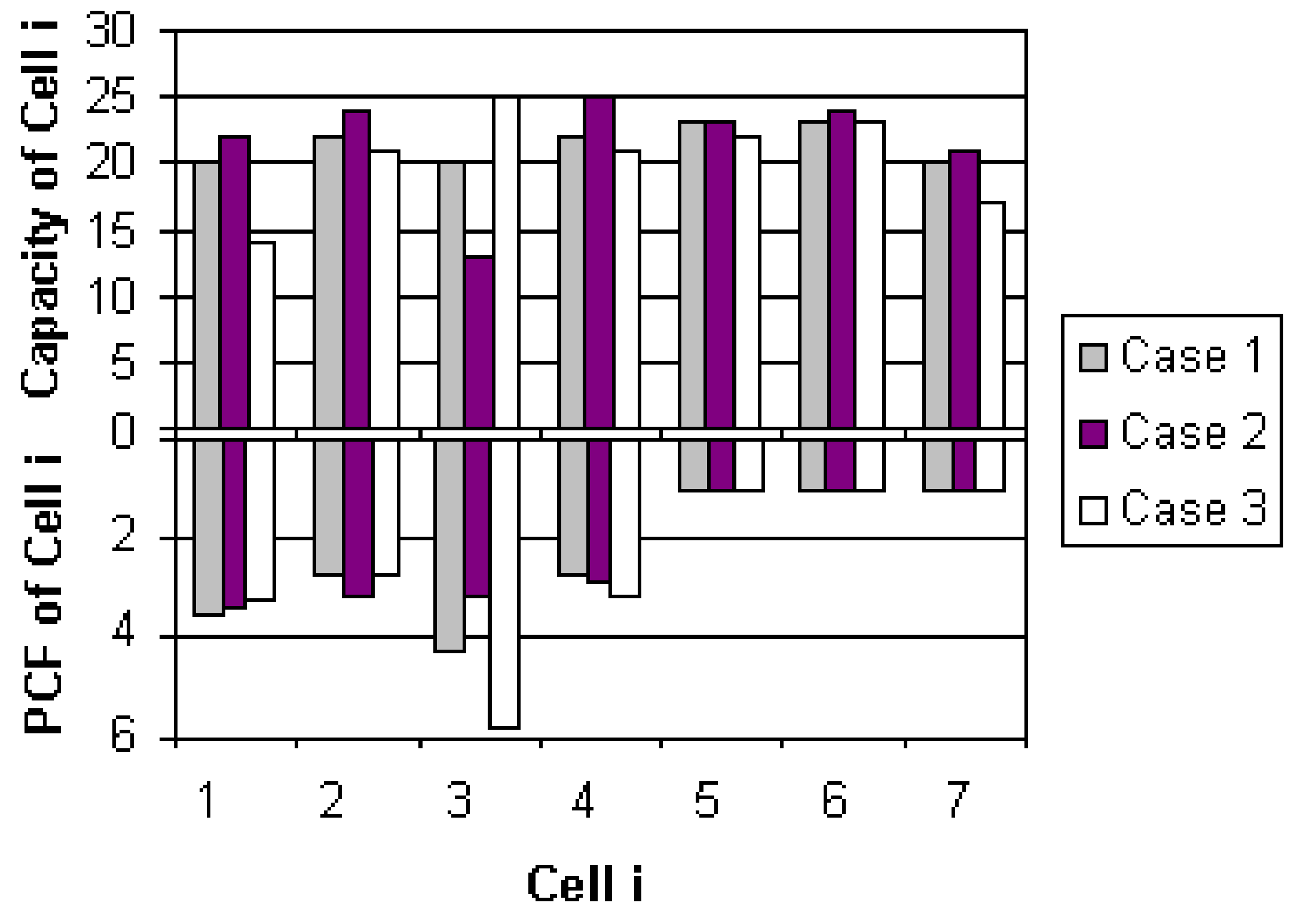




\section{Conclusions}

- Power Compensation Factor is a design tool to:

- Flexibly distribute the capacity allocation between cells

- Optimize the capacity of a network

- Maximize the capacity of a single cell

- Easy to implement in an existing network

- Does not require the relocation or addition of Base Stations. 


\section{Future Work}

- Pilot Power determines the cell geometry which determines the inter-cell and intra-cell interference

- Determine the sensitivity of capacity with respect to Pilot Power

- Determine the sensitivity of capacity with respect to Base Station location

- Complete Design: Location, forward power, reverse power. 